\title{
The prevalence of substance use disorders and substance use in anorexia nervosa: a systematic review and meta-analysis
}

Daniel J. Devoe ${ }^{1 *} \mathbb{D}$, Gina Dimitropoulos ${ }^{1,2}$, Alida Anderson ${ }^{1}$, Anees Bahji ${ }^{1}$, Jordyn Flanagan', Andrea Soumbasis ${ }^{1}$, Scott B. Patten ${ }^{1}$, Tom Lange ${ }^{1}$ and Georgios Paslakis ${ }^{3}$

\begin{abstract}
Aim: Individuals with anorexia nervosa (AN) often present with substance use and substance use disorders (SUDs). However, the prevalence of substance use and SUDs in AN has not been studied in-depth, especially the differences in the prevalence of SUDs between AN types [e.g., AN-R (restrictive type) and AN-BP (binge-eating/purge type]. Therefore, this systematic review and meta-analysis aimed to assess the prevalence of SUDs and substance use in AN samples.

Method: Systematic database searches of the peer-reviewed literature were conducted in the following online databases: MEDLINE, PsycINFO, Embase, and CINAHL from inception to January 2021. We restricted review eligibility to peer-reviewed research studies reporting the prevalence for either SUDs or substance use in individuals with AN. Random-effects meta-analyses using Freeman-Tukey double arcsine transformations were performed on eligible studies to estimate pooled proportions and 95\% confidence intervals (Cls).

Results: Fifty-two studies met the inclusion criteria, including 14,695 individuals identified as having AN (mean age: 22.82 years). Random pooled estimates showed that substance use disorders had a $16 \%$ prevalence in those with AN (AN-BP $=18 \%$ vs. AN-R=7\%). Drug abuse/dependence disorders had a prevalence of $7 \%$ in AN (AN-BP $=9 \%$ vs. $A N-R=5 \%)$. In studies that looked at specific abuse/dependence disorders, there was a 10\% prevalence of alcohol abuse/dependence in AN (AN-BP $=15 \%$ vs. AN-R $=3 \%$ ) and a $6 \%$ prevalence of cannabis abuse/dependence (AN$\mathrm{BP}=4 \% \mathrm{vs} . \mathrm{AN}-\mathrm{R}=0 \%$ ). In addition, in terms of substance use, there was a $37 \%$ prevalence for caffeine use, $29 \%$ prevalence for alcohol use, 25\% for tobacco use, and 14\% for cannabis use in individuals with AN.
\end{abstract}

Conclusion: This is the most comprehensive meta-analysis on the comorbid prevalence of SUDs and substance use in persons with AN, with an overall pooled prevalence of $16 \%$. Comorbid SUDs, including drugs, alcohol, and cannabis, were all more common in AN-BP compared to AN-R throughout. Therefore, clinicians should be aware of the high prevalence of SUD comorbidity and substance use in individuals with AN. Finally, clinicians should consider screening for SUDs and integrating treatments that target SUDs in individuals with AN.

Plain English Summary: Individuals with anorexia nervosa (AN) may also present with substance use or have a substance use disorder (SUDS). Thus, we conducted a systematic review and meta-analysis to determine the prevalence

\footnotetext{
*Correspondence: djadevoe@ucalgary.ca

'Department of Psychiatry, Cumming School of Medicine, Mathison

Centre for Mental Health Research and Education, University of Calgary,

3280 Hospital Drive NW, Calgary, AB T2N 4Z6, Canada

Full list of author information is available at the end of the article
} permits use, sharing, adaptation, distribution and reproduction in any medium or format, as long as you give appropriate credit to the original author(s) and the source, provide a link to the Creative Commons licence, and indicate if changes were made. The images or other third party material in this article are included in the article's Creative Commons licence, unless indicated otherwise in a credit line to the material. If material is not included in the article's Creative Commons licence and your intended use is not permitted by statutory regulation or exceeds the permitted use, you will need to obtain permission directly from the copyright holder. To view a copy of this licence, visit http://creativecommons.org/licenses/by/4.0/. The Creative Commons Public Domain Dedication waiver (http://creativeco mmons.org/publicdomain/zero/1.0/) applies to the data made available in this article, unless otherwise stated in a credit line to the data. 
of substance use and substance use disorders in individuals with AN. We examined published studies that reported the prevalence of either substance use or SUDs in individuals with AN. We found that substance use disorders had a $16 \%$ prevalence and that drug abuse/dependence disorders had a prevalence of $7 \%$ in those with AN. These rates were much higher in individuals with binge-eating/purging type compared to the restrictive AN. However, many specific substance use disorders and substance use types were low in individuals with AN. Nonetheless, clinicians should be aware of the high prevalence of SUD comorbidity and substance use in individuals with AN.

Keywords: Anorexia nervosa, Substance use disorders, Substance use, Eating disorders, Comorbidity, substance misuse, drug abuse/dependence

\section{Introduction}

Eating disorders (EDs) are associated with a series of comorbidities, including depression, anxiety, personality disorders, and substance use disorders (SUDs) [29]. A recently published meta-analysis on the prevalence rates examining the comorbidity of SUDs in EDs found that the pooled prevalence of SUDs in EDs was 22\% [6], with the prevalence of EDs among individuals seeking treatment for SUDs being 35\%. Thus, the prevalence of EDs in individuals with SUDs appears to be ten times higher than the prevalence of EDs in the general population [21], with the prevalence of SUDs among individuals with EDs in treatment between 25 and 50\% [22, 57].

Research shows weaker associations between restrictive types of EDs [e.g., Anorexia Nervosa (AN)] and SUDs, although mechanisms of addiction may also be at play in AN [26, 44, 60,61]. For example, cues such as pictures of underweight bodies or physical activities are reinforcers and are associated with activation/sensitization of brain structures of reward [24, 27], while other cues such as pictures of high-calorie foods do not go along with approach reactions [45]. Such findings have led to the "reward-centered" model, which posits that food cues are processed as aversive, but disorder-compatible signals are processed positively and activate the mesolimbic reward system [44]. Subsequently, restrictive eating behaviors and disorder-compatible behaviors in AN (e.g., fasting, physical activity, frequent weighing, etc.) acquire the character of automated habitual behaviors and may lead to maintenance of the disorder. Thus, comparable to addictive disorders, a transition from goal-directed to automatic habitual behaviors in response to disorder-compatible stimuli may be at play. In addition, innovative treatment approaches (such as repetitive transcranial magnetic stimulation and deep-brain stimulation), targeting brain activity associated with the regulation of both food and addictive substance intake, appear to be emerging and to show promising results [16, $28,48,49$ ], which may help with the reduction of symptoms in both AN and SUDs.

Overall, despite evidence demonstrating similarities in the underlying mechanisms, associations, and prevalence of AN and SUDs, SUD in AN have not been studied indepth in a systematic review and meta-analysis. In addition, information on the prevalence of substance use (at any frequency) in AN may help contextualize the specific patterns of substances that are more likely to lead to functional consequences in persons with AN. Therefore, this systematic review and meta-analysis aimed to: (1) assess the prevalence rates of comorbidities between AN and SUDs or substance use (2) assess the prevalence of SUDs and substance use by AN type (AN-R and AN-BP); and (3) assess the quality of peer-reviewed literature to date. This is necessary to understand AN comorbidities, clinical indicators, and outcomes and inform future treatment planning.

\section{Method \\ Protocol and guidelines}

This systematic review and meta-analysis were prospectively registered with PROSPERO and adhered to both PRISMA (preferred reporting for systematic reviews and meta-analyses) and MOOSE (meta-analysis of observational studies in epidemiology) recommendations [34, 39, 56].

\section{Systematic search strategy}

Systematic searches of the peer-reviewed literature was conducted following PRESS guidelines [47] in consultation with a medical librarian in four electronic databases (i.e., MEDLINE, PsycINFO, Embase, and CINAHL) from inception to October 13th, 2021. The key words included two concepts: (1) anorexia nervosa (AN) and (2) substance Use or substance use disorder terms. Database searches and an exhaustive list of key terms are provided in the Additional file 1: search material. Two blinded reviewers performed title/abstract screening (A.A. and J.F.) and full-text article screening (A.A. and A.S.) in duplicate. In addition, reference lists of included articles were hand-searched for other relevant studies.

\section{Study selection criteria}

Two reviewers selected peer-reviewed articles (A.A. and A.S.) for inclusion in this systematic review based on the 
following criteria: (1) research including participants with anorexia nervosa (AN), restrictive AN (AN-R), and $\mathrm{AN}$ of the binge-eating/purging type (AN-BP); and (2) reported on the prevalence of either substance use (e.g., alcohol use, tobacco use, cannabis use), substance use disorders, or drug abuse/misuse/dependence disorders. In addition, this review excluded studies that: (1) looked at the relationship between AN and other behavioral addictions (e.g., gambling disorder) or impulse control disorders, (2) study designs that were case reports, review articles, opinion pieces, and editorials, (3) studies that included use/abuse of prescribed medications and (4) did not report sufficient information to calculate a prevalence rate. Disagreements were first discussed in a consensus meeting, and D.D. decided on inclusion or exclusion.

\section{Data extraction}

Data extraction for Table 1 was completed in duplication (A.A. and G.P.), including the following study and participant characteristics: author, year of publication, country, study type, types of substance use/abuse/dependence, AN types, age (mean $\pm S D$ ), percent female (number of females), and outcomes reported. For the meta-analysis, the following data were extracted in duplicate (A.A. and D.D.): (1) author, (2) year of publication, (3) types of substance use, substance abuse/dependence disorders, and drug abuse/dependence disorders, (4) AN type, (5) numerator representing substance use/abuse/dependence disorders, and drug abuse/dependence disorders, (6) denominator representing AN sample, and (7) lifetime prevalence or period prevalence.

\section{Risk-of-bias assessment}

Studies included in this systematic review and meta-analysis were assessed for quality using a modified Downs and Black instrument [19] which contains 14-items for cross-sectional studies, providing a total score of 15 for each study indicating greater quality. Scores of $\geq 11.5$ (>75\%), 9-11 (60-74\%), and $<9(<60 \%)$ were taken to indicate high, moderate, and low quality, respectively.

\section{Data synthesis and analysis}

Due to potential heterogeneity between studies, successions of DerSimonian and Laird [18] random-effects meta-analyses were performed on eligible studies to estimate the pooled prevalence and 95\% CIs for substance use disorders, drug abuse/misuse/dependence disorders, and substance use. The primary outcome measure was total substance use disorders and the summary statistic used in the meta-analysis was the pooled prevalence. Many studies distinguish total substance use disorders from total drug abuse/dependence disorders by not including alcohol in the drug abuse/dependence disorder count. Thus, these two concepts were kept separate in the meta-analysis. In addition, differences in the pooled prevalence between AN-R and AN-BP were examined. All meta-analyses in this paper employed FreemanTukey double arcsine transformations, with the exact confidence interval method, by computing the weighted pooled estimate and then performing a back-transformation on the pooled estimate. This approach is favorable where there is zero count prevalence as it prevents these studies from being dropped from the meta-analysis, which would create a bias in prevalence estimates. Lifetime prevalence and period prevalence were first examined separately, but there was minimal variation between the two prevalence types. Thus, we combined the two (e.g., some studies reported lifetime and other studies reported period prevalence, both types were included in the same meta-analysis) to provide an overall prevalence for each outcome. Two studies was the minimum amount of studies included in each pooled meta-analysis. However, prevalence was also presented when reported by just one study, however this is not an estimate derived from a meta-analysis. Statistical heterogeneity was examined using the $\mathrm{I}^{2}$ statistics, an $\mathrm{I}^{2}$ value is only produced for a meta-analysis with four or more studies in the metaanalysis. We performed all analyses in STATA v.17 [54] and produced forest plots showing the prevalence of those with either substance use disorders or drug abuse/ dependence disorders in those with AN.

\section{Results}

\section{Search yield}

Database searches returned 2809 abstracts and titles. After duplicate references were removed, 2320 abstracts and titles were screened. The level of agreement between two blinded reviewers for screening was moderate $(\kappa=0.65)$. After resolution of discrepancies, eighty fulltext studies were retrieved and reviewed independently, of which a total of 52 studies met the inclusion criteria for this review (see Fig. 1). In total, 35 studies measured SUDs, 17 measured substance use, with six of these studies measuring both SUDs and substance use.

\section{Participant characteristics and study characteristics}

There was a total 14,695 individuals identified as having AN included in this review, ranging from sample sizes of 15 to 8069 for individuals with AN in separate studies, Table 1 . The mean age of individuals with AN was 22.82 years (Range 14.3-35.0), and the percentage of females was $95.6 \%$.

Studies were published between 1983 and 2019. Most studies were conducted in North America $(n=26)$, followed by Europe $(n=16)$, Asia $(n=6)$, and New Zealand 
Table 1 Details of Included Studies ( $n=52)$

\begin{tabular}{|c|c|c|c|c|c|c|c|}
\hline \multirow[t]{2}{*}{ Study } & \multirow[t]{2}{*}{ Year } & \multirow[t]{2}{*}{ Country } & \multirow{2}{*}{$\begin{array}{l}\text { Type(s) of SUDs or } \\
\text { substance use }\end{array}$} & \multirow[t]{2}{*}{ Type(s) of AN } & \multicolumn{3}{|l|}{ AN patients } \\
\hline & & & & & $\mathbf{N}$ & Age (Mean \pm SD) & $\%$ Female \\
\hline Anzengruber et al. & 2006 & USA & Smoking (nicotine) & $\begin{array}{l}\text { AN-R, AN-P, AN-B, } \\
\text { ANBN }\end{array}$ & $\begin{array}{l}A N-R=306 \\
A N-P=186 \\
A N-B=107 \\
\text { ANBN }=180\end{array}$ & $\begin{array}{l}\text { Initial sample } \\
(n=897): 26.3 \pm 8.3\end{array}$ & $100 \%$ \\
\hline Blinder et al. & 2006 & USA & $\begin{array}{l}\text { Alcohol, cannabis, } \\
\text { polysubstance, } \\
\text { other substance, } \\
\text { amphetamine, } \\
\text { sedative/hypnotic/ } \\
\text { anxiolytic, cocaine, } \\
\text { hallucinogen, } \\
\text { opioid, inhalant } \\
\text { abuse/ dependen- } \\
\text { cies }\end{array}$ & $A N-R, A N-B$ & $\begin{array}{l}A N-R=520 \\
A N-B=436\end{array}$ & $\begin{array}{l}A N-R=20.9 \pm 9.1 \\
A N-B=23.8 \pm 8.5\end{array}$ & $100 \%$ \\
\hline Bodell et al. & 2013 & USA & SUD & AN & 30 & $\begin{array}{l}\text { Total sample: } 35 \\
\pm 9\end{array}$ & $100 \%$ \\
\hline Braun et al. & 1994 & USA & $\begin{array}{l}\text { Alcohol/substance } \\
\text { dependence }\end{array}$ & $A N-R$ & 34 & 24.8 & $100 \%$ \\
\hline Bulik et al. & 1992 & USA & $\begin{array}{l}\text { Licit and illicit } \\
\text { substance use }\end{array}$ & AN & 27 & $20.3 \pm 10.5$ & $100 \%$ \\
\hline Bulik et al & 2008 & USA & $\begin{array}{l}\text { Psychoactive sub- } \\
\text { stance abuse and } \\
\text { dependence }\end{array}$ & $\begin{array}{l}\text { AN-R, AN-P, AN-B, } \\
\text { ANBN }\end{array}$ & 432 & $30.4 \pm 11.3$ & $\begin{array}{l}95 \% \\
(n=410)\end{array}$ \\
\hline Burgalassi et al. & 2009 & Italy & Caffeine & $A N-R, A N-B P$ & 15 & $26 \pm 5$ & $100 \%$ \\
\hline Carlat et al. & 1997 & USA & Substance abuse & AN & 30 & $\begin{array}{l}\text { At onset: } \\
19.0 \pm 5.6 \\
\text { At first treatment: } \\
20.3 \pm 6.0\end{array}$ & $0 \%$ \\
\hline Casper and Jabine & 1996 & USA & $\begin{array}{l}\text { Alcohol abuse/ } \\
\text { dependence, drug } \\
\text { abuse/depend- } \\
\text { ence, tobacco } \\
\text { dependence }\end{array}$ & $\begin{array}{l}\text { AN-R, } \\
\text { AN-P }\end{array}$ & 75 & $\begin{array}{l}\text { Early Adolescent } \\
\text { Onset: } \\
16.2 \pm 3.3 \\
\text { Late Adolescent } \\
\text { Onset: } \\
19.7 \pm 3.3 \\
\text { Adult Onset: } \\
25.2 \pm 3.7\end{array}$ & $100 \%$ \\
\hline Corbridge and Bell & 1996 & UK & $\begin{array}{l}\text { Alcohol and drug } \\
\text { misuse }\end{array}$ & AN & 25 & $\begin{array}{l}\text { Age of onset: } \\
23.2\end{array}$ & $\begin{array}{l}\text { Total sample: } \\
97 \% \\
(n=125)\end{array}$ \\
\hline Corcos et al. & 2001 & $\begin{array}{l}\text { France, } \\
\text { Switzerland, } \\
\text { Belgium }\end{array}$ & $\begin{array}{l}\text { Alcohol, drug, } \\
\text { and psychotropic } \\
\text { consumption }\end{array}$ & $\begin{array}{l}\text { AN-R, } \\
\text { AN-P }\end{array}$ & $\begin{array}{l}A N-R=111 \\
A N-P=55\end{array}$ & $\begin{array}{l}A N-R=19.3 \\
A N-P=20.6\end{array}$ & $100 \%$ \\
\hline Deter and Herzog & 1994 & Germany & Substance abuse & $A N-R, A N-P$ & $\begin{array}{l}A N-R=29 \\
A N-P=55\end{array}$ & $\begin{array}{l}\text { Presentation: } \\
20.7 \pm 6.0 \\
\text { Follow-up: } \\
32.5 \pm 6.1\end{array}$ & $100 \%$ \\
\hline Eddy et al. & 2002 & USA & $\begin{array}{l}\text { Drug and alcohol } \\
\text { abuse }\end{array}$ & $A N-R, A N-B P$ & $\begin{array}{l}A N-R=51 \\
A N-B P=85\end{array}$ & $\begin{array}{l}\text { AN-R (no B/P } \\
\text { history) }=20.8 \\
\text { AN-R (history } \\
\text { of B/P) }=23.8 \\
\text { AN-BP }=22.7\end{array}$ & $100 \%$ \\
\hline Fairburn et al. & 1999 & UK & $\begin{array}{l}\text { Drug and alcohol } \\
\text { abuse }\end{array}$ & AN & 67 & $22.4 \pm 4.8$ & $100 \%$ \\
\hline $\begin{array}{l}\text { Fichter and } \\
\text { Quadlieg }\end{array}$ & 1999 & Germany & $\begin{array}{l}\text { Substance abuse } \\
\text { (including alcohol } \\
\text { and tranquilizers) }\end{array}$ & $A N-R, A N-B P$ & $\begin{array}{l}A N-R=30 \\
A N-B P=73\end{array}$ & $\begin{array}{l}\text { Upon admission: } \\
24.9 \pm 6.7\end{array}$ & $100 \%$ \\
\hline Fichter et al. & 2006 & Germany & $\begin{array}{l}\text { Substance abuse } \\
\text { (including drug } \\
\text { dependence) }\end{array}$ & $A N-R, A N-B P$ & $\begin{array}{l}A N-R=30 \\
A N-B P=73\end{array}$ & $\begin{array}{l}\text { Upon admission: } \\
24.9 \pm 6.7\end{array}$ & $100 \%$ \\
\hline
\end{tabular}


Table 1 (continued)

\begin{tabular}{|c|c|c|c|c|c|c|c|}
\hline \multirow[t]{2}{*}{ Study } & \multirow[t]{2}{*}{ Year } & \multirow[t]{2}{*}{ Country } & \multirow{2}{*}{$\begin{array}{l}\text { Type(s) of SUDs or } \\
\text { substance use }\end{array}$} & \multirow{2}{*}{ Type(s) of AN } & \multicolumn{3}{|l|}{ AN patients } \\
\hline & & & & & $\mathrm{N}$ & Age (Mean \pm SD) & $\%$ Female \\
\hline Fioravanti et al. & 2014 & Italy & $\begin{array}{l}\text { Cocaine and } \\
\text { amphetamine } \\
\text { abuse }\end{array}$ & $\begin{array}{l}\text { AN-R, } \\
\text { AN-BP }\end{array}$ & $\begin{array}{l}A N-R=28 \\
A N-B P=35\end{array}$ & $\begin{array}{l}\text { AN-R: } \\
25.93 \pm 8.94 \\
\text { AN-B/P: } \\
25.77 \pm 8.80\end{array}$ & $100 \%$ \\
\hline Franko et al. & 2005 & USA & $\begin{array}{l}\text { Alcohol Use Disor- } \\
\text { der (AUD) }\end{array}$ & $A N-R, A N-B P$ & $\begin{array}{l}A N-R=51 \\
A N-B P=85\end{array}$ & $\begin{array}{l}\text { Not reported } \\
\text { (Inclu- } \\
\text { sion:> } 12 \text { years) }\end{array}$ & $100 \%$ \\
\hline Franko et al. & 2008 & USA & $\begin{array}{l}\text { Drug Use Disorder } \\
\text { (DUD) }\end{array}$ & $A N-R, A N-B P$ & $\begin{array}{l}A N-R=51 \\
A N-B P=85\end{array}$ & $\begin{array}{l}\text { Entire sample at } \\
\text { entry: } \\
24.68 \pm 6.7\end{array}$ & $100 \%$ \\
\hline George and Waller & 2005 & UK & Smoking/nicotine & AN & 25 & $31.2 \pm 2.16$ & $100 \%$ \\
\hline Hall et al. & 1984 & New Zealand & $\begin{array}{l}\text { Alcohol Abuse } \\
\text { Disorder }\end{array}$ & AN & 50 & $\begin{array}{l}\text { Onset: } \\
16.2 \pm 2.7 \\
\text { Presentation: } \\
20.1 \pm 5.6\end{array}$ & $100 \%$ \\
\hline Haug et al. & 2001 & USA & $\begin{array}{l}\text { Tobacco, caffeine, } \\
\text { alcohol, marijuana, } \\
\text { other drug use } \\
\text { (cocaine, opiates, } \\
\text { hallucinogens) }\end{array}$ & $A N-R, A N-P$ & $\begin{array}{l}A N-R=34 \\
A N-P=31\end{array}$ & $\begin{array}{l}\text { Total sample: } \\
26.2 \pm 11.5\end{array}$ & $100 \%$ \\
\hline Henzel & 1984 & USA & Alcoholism & AN & 15 & 24 (median) & $\begin{array}{l}80 \% \\
(n=12)\end{array}$ \\
\hline Herzog et al. & 1992 & USA & SUD & $\begin{array}{l}\text { AN, } \\
\text { ANBN }\end{array}$ & $\begin{array}{l}\mathrm{AN}=41 \\
\mathrm{ANBN}=90\end{array}$ & $\begin{array}{l}\mathrm{AN}=19.1 \pm 6.4 \\
\mathrm{ANBN}=17.5 \pm 4.6\end{array}$ & $100 \%$ \\
\hline Herzog et al. & 1999 & USA & SUD & $A N-R, A N-B P$ & $\begin{array}{l}A N-R=51 \\
A N-B P=85\end{array}$ & $\begin{array}{l}A N-R=23.9 \pm 8.5 \\
A N-B P=24.5 \pm 5.9\end{array}$ & $100 \%$ \\
\hline Herzog et al. & 2006 & USA & $\begin{array}{l}\text { DUD; narcotics, } \\
\text { amphetamines, } \\
\text { cocaine, sedatives, } \\
\text { marijuana, LSD, } \\
\text { solvents, polydrug } \\
\text { use }\end{array}$ & $A N-R, A N-B P$ & $\begin{array}{l}A N-R=51 \\
A N-B P=85\end{array}$ & $\begin{array}{l}\text { Not reported } \\
\text { (Inclu- } \\
\text { sion:>12 years) }\end{array}$ & $100 \%$ \\
\hline Hudson et al. & 1983 & USA & $\begin{array}{l}\text { Alcohol abuse/ } \\
\text { dependence, } \\
\text { Amphetamine } \\
\text { abuse or depend- } \\
\text { ence, } \\
\text { Other substance } \\
\text { use }\end{array}$ & $\begin{array}{l}\text { AN } \\
\text { ANBN }\end{array}$ & $\begin{array}{l}\mathrm{AN}=16 \\
\mathrm{ANBN}=25\end{array}$ & $\begin{array}{l}\text { AN }=25.0 \pm 7.0(15 \\
\text { Females only, one } \\
\text { male aged } 24) \\
\text { ANBN=25.8 } \pm 7.3 \\
\text { (24 females only, } \\
\text { one man aged } 28 \text { ) }\end{array}$ & $95.1 \%(n=39)$ \\
\hline Iwasaki et al. & 2000 & Japan & $\begin{array}{l}\text { Alcohol abuse, } \\
\text { Sedative abuse, } \\
\text { Inhalant abuse }\end{array}$ & $\begin{array}{l}\text { AN-R } \\
\text { AN-BP }\end{array}$ & $\begin{array}{l}A N-R=62 \\
A N-B P=36\end{array}$ & $\begin{array}{l}A N-R=21.3 \pm 5.5 \\
A N-B P=24.2 \pm 4.7\end{array}$ & $100 \%$ \\
\hline Jordan et al. & 2003 & New Zealand & $\begin{array}{l}\text { Alcohol abuse/ } \\
\text { dependence, } \\
\text { cannabis abuse/ } \\
\text { dependence, psy- } \\
\text { choactive SUD }\end{array}$ & $A N-R, A N-B P$ & $\begin{array}{l}A N-R=24 \\
A N-B P=16\end{array}$ & $23.15 \pm 6.69$ & $100 \%$ \\
\hline Jordan et al. & 2008 & New Zealand & $\begin{array}{l}\text { Alcohol abuse/ } \\
\text { dependence, } \\
\text { cannabis abuse/ } \\
\text { dependence, any } \\
\text { psychoactive SUD }\end{array}$ & $A N-R, A N-B P$ & $\begin{array}{l}A N-R=31 \\
A N-B P=25\end{array}$ & 20.5 & $100 \%$ \\
\hline Kask et al. & 2016 & Sweden & $\begin{array}{l}\text { Alcohol use disor- } \\
\text { der, other SUD }\end{array}$ & AN & 8069 & $19.5 \pm 6.2$ & $100 \%$ \\
\hline Kask et al & 2017 & Sweden & $\begin{array}{l}\text { Alcohol use } \\
\text { disorder, other } \\
\text { substance use } \\
\text { disorder }\end{array}$ & AN & 609 & $\begin{array}{l}\text { Mean age of first } \\
\text { hospitalization: } \\
18.2 \pm 6.9\end{array}$ & $0 \%$ \\
\hline
\end{tabular}


Table 1 (continued)

\begin{tabular}{|c|c|c|c|c|c|c|c|}
\hline \multirow[t]{2}{*}{ Study } & \multirow[t]{2}{*}{ Year } & \multirow[t]{2}{*}{ Country } & \multirow{2}{*}{$\begin{array}{l}\text { Type(s) of SUDs or } \\
\text { substance use }\end{array}$} & \multirow{2}{*}{ Type(s) of AN } & \multicolumn{3}{|l|}{ AN patients } \\
\hline & & & & & $\mathrm{N}$ & Age (Mean \pm SD) & $\%$ Female \\
\hline Kirkpatrick et al. & 2019 & Canada & Substance Use & $\begin{array}{l}\text { AN-R, AN-BP, atypi- } \\
\text { cal AN }\end{array}$ & $\begin{array}{l}\mathrm{AN}-\mathrm{R}=40 \\
\mathrm{AN}-\mathrm{BP}=19 \\
\text { Atypical } \mathrm{AN}=14\end{array}$ & $\begin{array}{l}\text { SUG (all ED): } \\
16.3 \pm 1.0 \\
\text { NSUG (all ED): } \\
14.3 \pm 2.9\end{array}$ & $\begin{array}{l}\text { SUG (all ED) } \\
97.6 \% \\
\text { NSUG (all ED) } \\
81.7 \%\end{array}$ \\
\hline Krahn et al. & 1991 & USA & $\begin{array}{l}\text { Caffeine consump- } \\
\text { tion }\end{array}$ & $\begin{array}{l}\text { AN, } \\
\text { ANBN }\end{array}$ & $\begin{array}{l}\mathrm{AN}=14 \\
\mathrm{ANBN}=11\end{array}$ & $\begin{array}{l}\text { Total sample: } \\
24.7\end{array}$ & N/A \\
\hline Krug et al. & 2008 & $\begin{array}{l}\text { Spain, } \\
\text { UK, } \\
\text { Italy, } \\
\text { Austria, } \\
\text { Slovenia }\end{array}$ & $\begin{array}{l}\text { Tobacco, alcohol, } \\
\text { and drug use }\end{array}$ & $A N-R, A N-B P$ & $\begin{array}{l}A N-R=172 \\
A N-B P=156\end{array}$ & $\begin{array}{l}\text { All EDs: } \\
27.2 \pm 8.9\end{array}$ & $\begin{array}{l}\text { All EDs: } \\
96.6 \% \\
(n=849)\end{array}$ \\
\hline Laessle et al. & 1989 & Germany & SUD & AN-R, AN-BP & $\begin{array}{l}A N-R=21 \\
A N-B P=20\end{array}$ & $\begin{array}{l}\mathrm{AN}-\mathrm{R}=20.9 \pm 4.1 \\
\mathrm{AN}-\mathrm{BP}=22.1 \pm 4.2\end{array}$ & $100 \%$ \\
\hline Machado et al. & 2004 & Portugal & $\begin{array}{l}\text { Alcohol and drug } \\
\text { abuse }\end{array}$ & $A N-R, A N-B P$ & $\begin{array}{l}A N-R=42 \\
A N-B P=23\end{array}$ & $16.8 \pm 13.4$ & $100 \%$ \\
\hline Mann et al. & 2014 & USA & $\begin{array}{l}\text { Substance use } \\
\text { (alcohol, cannabis, } \\
\text { tobacco, and any } \\
\text { other substance) }\end{array}$ & AN & 118 & $\begin{array}{l}\text { Total sample: } \\
15.77 \pm 1.84\end{array}$ & Total sample: $90.7 \%$ \\
\hline Milos et al. & 2003 & Switzerland & $\begin{array}{l}\text { Substance related } \\
\text { disorders (use and } \\
\text { dependency) }\end{array}$ & AN & 77 & $24.7 \pm 5.6$ & $100 \%$ \\
\hline Nagata et al. & 2000 & Japan & $\begin{array}{l}\text { Alcohol and Illicit } \\
\text { drug (heroin, } \\
\text { amphetamines, } \\
\text { other psychoactive } \\
\text { substances) use }\end{array}$ & $A N-R, A N-B P$ & $\begin{array}{l}A N-R=60 \\
A N-B P=62\end{array}$ & $\begin{array}{l}A N-R=22.3 \pm 4.0 \\
A N-B P=25.0 \pm 5.1\end{array}$ & $100 \%$ \\
\hline Nagata et al. & 2002 & Japan & DUD & $A N-R, A N-B P$ & $\begin{array}{l}\mathrm{AN}-\mathrm{R}=62 \\
\mathrm{AN}-\mathrm{BP}=48\end{array}$ & $\begin{array}{l}\text { ED and DUD: } \\
25.1 \pm 5.7 \\
\text { ED without DUD: } \\
24.2 \pm 5.5\end{array}$ & $100 \%$ \\
\hline Nagata et al. & 2003 & Japan & $\begin{array}{l}\text { Drugs other than } \\
\text { alcohol }\end{array}$ & $A N-B P, A N-R$ & $\begin{array}{l}A N-B P=8 \\
A N-R=2\end{array}$ & $\begin{array}{l}\text { Total ED + DUD } \\
\text { group: } 24.8 \pm 5.4\end{array}$ & $100 \%$ \\
\hline Nozoe et al. & 1995 & Japan & $\begin{array}{l}\text { Stimulant (alcohol, } \\
\text { coffee, cigarettes) } \\
\text { abuse }\end{array}$ & AN & 55 & $\begin{array}{l}\text { Total sample at } \\
\text { admission: } 20.0\end{array}$ & $90.9 \%(n=50)$ \\
\hline Selby et al. & 1995 & USA & $\begin{array}{l}\text { Substance misuse } \\
\text { problems }\end{array}$ & AN & 25 & $\begin{array}{l}\text { Entire ED sample: } \\
30.07 \pm 13.78\end{array}$ & $100 \%$ \\
\hline Strober et al. & 1995 & USA & $\begin{array}{l}\text { Alcohol, cocaine, } \\
\text { amphetamines, } \\
\text { cannabis, polysub- } \\
\text { stance use/abuse }\end{array}$ & $A N-R, A N-B$ & $\begin{array}{l}A N-R=77 \\
A N-B=18\end{array}$ & $\begin{array}{l}15.1 \text { (SD not } \\
\text { reported) }\end{array}$ & $94 \%(n=89)$ \\
\hline Sullivan et al. & 1998 & New Zealand & $\begin{array}{l}\text { Alcohol, cannabis, } \\
\text { other drug, and any } \\
\text { drug dependence }\end{array}$ & AN & 70 & $\begin{array}{l}\text { At admission: } \\
20.9 \pm 8.0 \\
\text { Interview: } \\
32.4 \pm 7.8\end{array}$ & $100 \%$ \\
\hline Tanaka et al. & 2001 & Japan & Alcohol abuse & $A N-R, A N-B P$ & $\begin{array}{l}A N-R=27 \\
A N-B P=34\end{array}$ & $\begin{array}{l}\text { At first referral: } \\
22.7 \pm 6.0 \text { years }\end{array}$ & $100 \%$ \\
\hline Toner et al. & 1986 & Canada & $\begin{array}{l}\text { SUD; alcohol, drug, } \\
\text { and tobacco abuse }\end{array}$ & $A N-R, A N-B$ & $\begin{array}{l}A N-R=30 \\
A N-B=25\end{array}$ & $\begin{array}{l}A N-R=28.0 \pm 5.3 \\
A N-B=28.2 \pm 4.0\end{array}$ & $100 \%$ \\
\hline Ulfvebrand et al. & 2015 & Sweden & $\begin{array}{l}\text { Alcohol depend- } \\
\text { ence, } \\
\text { Alcohol abuse, } \\
\text { Substance depend- } \\
\text { ence, } \\
\text { Substance abuse, } \\
\text { Substance use } \\
\text { disorders }\end{array}$ & $\begin{array}{l}\text { AN-R } \\
\text { AN-BP }\end{array}$ & $\begin{array}{l}A N-R=926 \\
A N-B P=466\end{array}$ & $\begin{array}{l}\text { AN-R (female) }=24 \\
\text { AN-R (male) }=23 \\
\text { AN-BP } \\
\text { (female) }=24 \\
\text { AN-BP (male) }=21 \\
\text { (SD not reported) }\end{array}$ & $\begin{array}{l}96.7 \% \\
(n=6921)\end{array}$ \\
\hline
\end{tabular}


Table 1 (continued)

\begin{tabular}{|c|c|c|c|c|c|c|c|}
\hline \multirow[t]{2}{*}{ Study } & \multirow[t]{2}{*}{ Year } & \multirow[t]{2}{*}{ Country } & \multirow{2}{*}{$\begin{array}{l}\text { Type(s) of SUDs or } \\
\text { substance use }\end{array}$} & \multirow{2}{*}{ Type(s) of AN } & \multicolumn{3}{|l|}{ AN patients } \\
\hline & & & & & $\mathrm{N}$ & Age $($ Mean $\pm S D)$ & $\%$ Female \\
\hline $\begin{array}{l}\text { Wiederman and } \\
\text { Pryor }\end{array}$ & 1996A & USA & $\begin{array}{l}\text { Alcohol, ampheta- } \\
\text { mines, barbiturates, } \\
\text { hallucinogens, } \\
\text { marijuana, tran- } \\
\text { quilizers, cocaine, } \\
\text { cigarettes }\end{array}$ & AN & 134 & $\begin{array}{l}\text { Total sample: } \\
24.15 \pm 7.69\end{array}$ & $100 \%$ \\
\hline $\begin{array}{l}\text { Wiederman and } \\
\text { Pryor }\end{array}$ & 1996B & USA & $\begin{array}{l}\text { Alcohol, ampheta- } \\
\text { mines, barbiturates, } \\
\text { hallucinogens, } \\
\text { marijuana, tran- } \\
\text { quilizers, cocaine, } \\
\text { cigarettes }\end{array}$ & $A N-R, A N-B P$ & $\begin{array}{l}A N-R=46 \\
A N-B P=13\end{array}$ & $\begin{array}{l}\text { Total sample: } \\
15.44 \pm 1.35\end{array}$ & $100 \%$ \\
\hline Wiseman et al. & 1998 & USA & $\begin{array}{l}\text { Cigarettes (nico- } \\
\text { tine) }\end{array}$ & $A N-R, A N-B P$ & $\begin{array}{l}\mathrm{AN}-\mathrm{R}=56 \\
\mathrm{AN}-\mathrm{BP}=12 \\
\text { (included with BN } \\
\text { group) }\end{array}$ & $\begin{array}{l}\text { Total ED group: } \\
15.35 \pm 1.4\end{array}$ & $100 \%$ \\
\hline
\end{tabular}

$A N-R$ Anorexia nervosa-restricting type; $A N-P$ anorexia nervosa-purging type; $A N-B$ anorexia nervosa-binging type; $A N B N$ anorexia nervosa and bulimia nervosa; $E D$ eating disorder; $A N-B P$ anorexia nervosa, binge-eating/purging type; $B N$ bulimia nervosa; $M D D$ major depressive disorder; $A U D$ alcohol use disorder; $D U D$ drug use disorder; SUG substance use group; NSUG no substance use group

$(n=4)$. Twenty-two studies included a comparison or control group in their respective study. Forty-two studies identified individuals with AN from a hospital setting $(9=$ outpatient, 7 =inpatient), ED programs, or specialized clinic, with six studies identifying AN individuals from research studies. Additional file 1: Table S2 describes in greater detail outcomes reported in each study, including subgroup analyses, comparison groups, sex differences, and other relevant information related to AN and SUDs or substance use outcomes.

\section{Quality assessment of included studies}

All studies included in this systematic review were evaluated with the modified Downs and Black instrument (Additional file 1: Table S1). The average Downs and Black score was $10.9 / 15$, demonstrating mostly moderate quality across studies. The majority of studies clearly described their main aims, measures, and findings. However, most studies included in this review failed to account for the effects of significant covariates.

\section{Prevalence of substance use disorders and drug abuse/ dependence}

In studies that looked at total substance use disorders, random pooled estimates demonstrated that substance use disorders had a $16 \%$ prevalence in those with AN (95\% $\mathrm{CI}=0.11-0.20 ; \mathrm{I}^{2}=86.3 \% ; 15$ studies, $\mathrm{N}=3118$ ), see Fig. 2. Individuals with AN-BP had a higher prevalence of substance use disorders at $18 \%$ (95\% CI $=0.12-$ $0.26 ; \mathrm{I}^{2}=82.1 \%$; 6 studies, $\mathrm{N}=1058$ ) compared to $7 \%$ in those with AN-R (95\% CI =0.04-0.10; $\mathrm{I}^{2}=69.3 \%$; 8 studies, $\mathrm{N}=1635$ ), see Additional file 1: Figure S1.

Drug abuse/dependence disorders had a prevalence of $7 \%$ in $\mathrm{AN}$ (95\% CI=0.05-0.09; $\mathrm{I}^{2}=81.1 \% ; 13$ studies, $\mathrm{N}=10,443$ ), with individuals with $\mathrm{AN}-\mathrm{BP}$ having a higher prevalence of drug abuse/dependence at $9 \%$ (95\% $\mathrm{CI}=0.03-0.17 ; \mathrm{I}^{2}=65.8 \%$; 5 studies, $\mathrm{N}=235$ ) compared to $5 \%$ in those with AN-R $(95 \% \mathrm{CI}=0.02-0.09$;; $\mathrm{I}^{2}=35.8 \% ; 5$ studies, $\mathrm{N}=278$ ), see Additional file 1: figure S2.

\section{Prevalence of specific substance use disorders}

In studies that looked at specific substance use disorders, random pooled estimates demonstrated that there was a $10 \%$ prevalence of alcohol abuse/dependence in AN (15\% AN-BP vs. 3\% AN-R), 6\% prevalence of cannabis abuse/ dependence in AN (4\% AN-BP vs. 0\% AN-R), and a 5\% prevalence of amphetamine abuse/dependence in AN. However, the majority of specific substance use disorders identified in this review remained low comparatively. Prevalence estimates for other specific forms of substance abuse/dependence are provided in Table 2.

\section{Prevalence of substance use}

There was a $20 \%$ prevalence for substance use $(95 \%$ $\mathrm{CI}=0.08-0.34 ; \mathrm{I}^{2}=94.27 ; 4$ studies, $\mathrm{N}=769$ ) in studies that looked at substance use total in individuals with AN. In studies that looked at types of substance use in individuals with AN, there was a $37 \%$ prevalence for caffeine $(95 \% \mathrm{CI}=0.08-0.73 ; 3$ studies, $\mathrm{N}=107$ ), followed by a $29 \%$ prevalence for alcohol $(95 \% \mathrm{CI}=0.22-0.36$; 

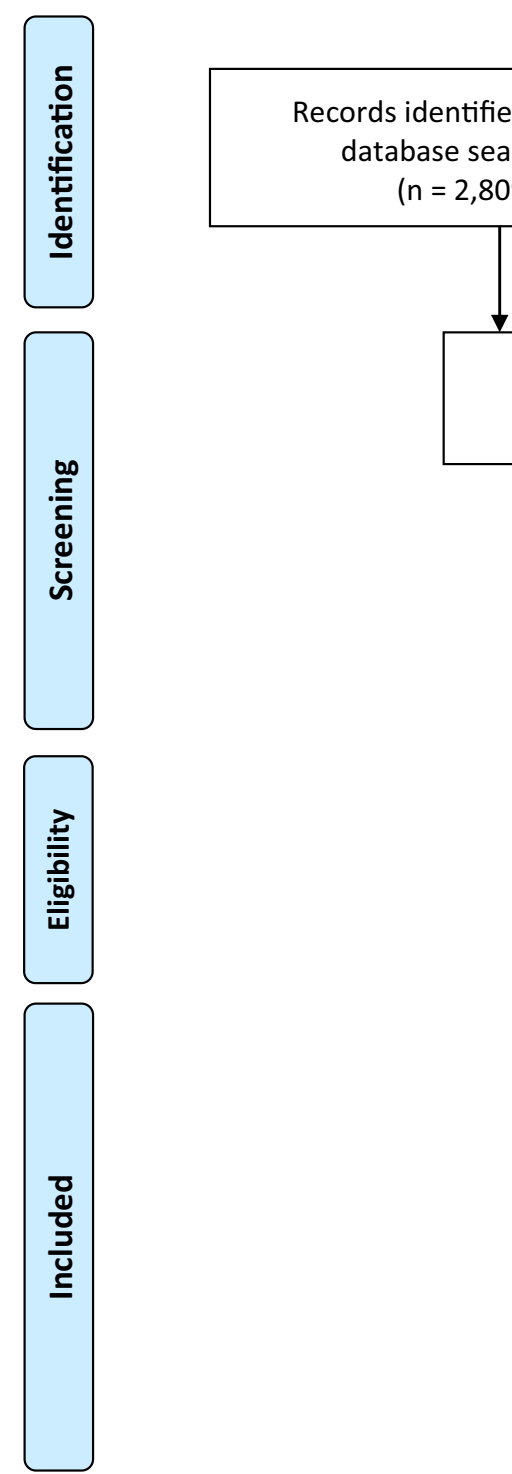

Records after duplicates removed
\[ (\mathrm{n}=2,320) \]

Additional records identified through other sources $(n=16)$

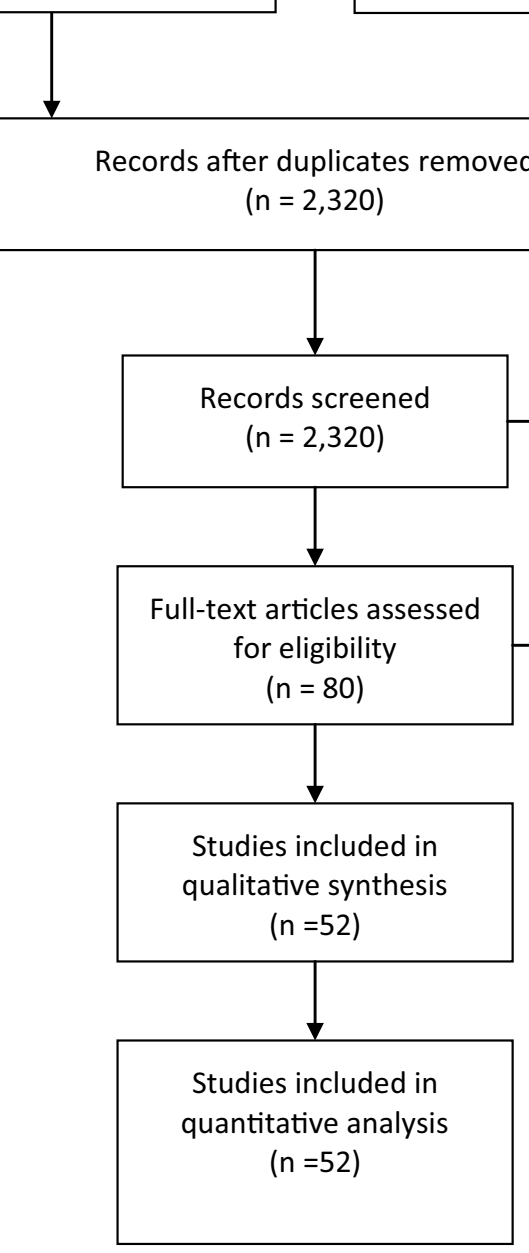

Fig. 1 PRISMA flow diagram

$\mathrm{I}^{2}=70.56 ; 5$ studies, $\left.\mathrm{N}=677\right), 25 \%$ for tobacco $(95 \%$ $\mathrm{CI}=0.16-0.34 ; \mathrm{I}^{2}=90.65 ; 9$ studies, $\left.\mathrm{N}=1352\right), \quad 14 \%$ for cannabis $\left(95 \% \mathrm{CI}=0.03-0.28 ; \mathrm{I}^{2}=95.18 ; 5\right.$ studies, $\mathrm{N}=720)$, and $14 \%$ stimulants use $(95 \% \mathrm{CI}=0.11-0.18$; 2 studies, $\mathrm{N}=383$ ). However, the majority of specific substance use identified in this review remained low comparatively. Prevalence estimates for other forms of substance use are provided in Table 3.

\section{Discussion}

\section{Summary of findings}

The present meta-analysis evaluated the prevalence of co-occurring substance use and substance use disorders (SUD) among persons with anorexia nervosa
(AN). In total, 52 studies met review eligibility criteria. The overall prevalence of substance use of any kind was $20 \%$, including caffeine (37\%), alcohol (29\%), tobacco $(25 \%)$, cannabis $(14 \%)$, and stimulants $(14 \%)$. The overall prevalence of any SUD was $16 \%$, including drug abuse/dependence (7\%), alcohol (10\%), cannabis (6\%), and amphetamines (5\%). Globally, the prevalence of drug use disorders among the population aged 15-64 is estimated to be $0.71 \%$ (Drugs \& Crime, 2019), thus the prevalence in AN appears to be high comparatively. The sample included in this meta-analysis was predominantly female, however, when looking at studies that included only males in the current review, males had an estimated prevalence of $17 \%$ for SUD total, $6 \%$ for 


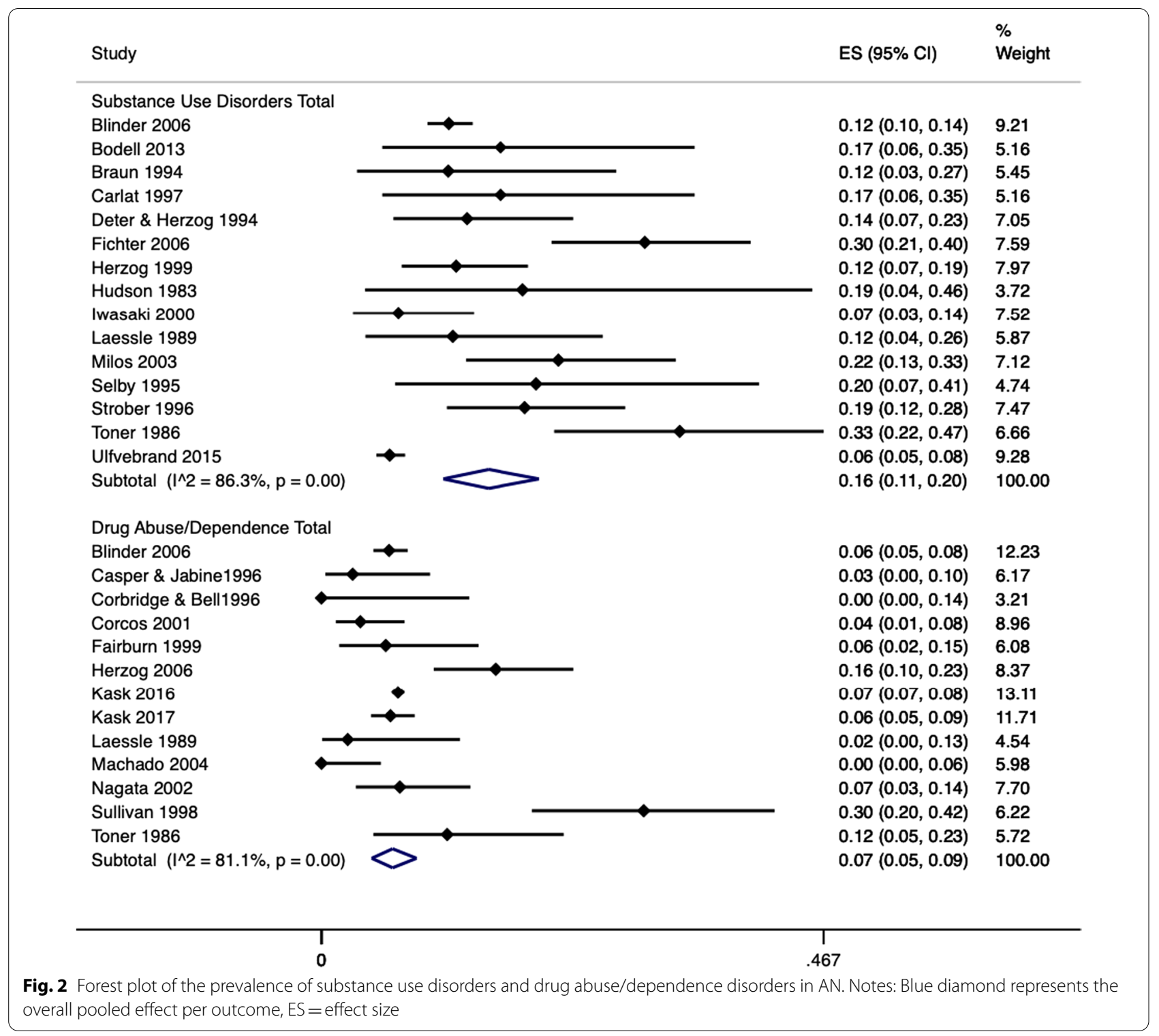

drug abuse/dependence, and between a $8-13 \%$ prevalence for alcohol abuse/dependence, which appears to be similar to females but based on very few studies. The overall sample was also younger in this meta-analysis with a mean age of 23 , and potentially overtime the prevalence of these disorders may increase. Substance use and SUD prevalence were higher among people with the binge-eating/purge (AN-BP) type than those with the restrictive (AN-R) type throughout. However, the majority of SUDs and substance use appear to be very low or not present in AN patients including sedative use, hallucinogen use, opioids, and inhalants.

\section{Treatment implications}

The high prevalence of comorbid substance use and SUD in persons with $\mathrm{AN}$ has important treatment implications. SUD management in the absence of ED comorbidity begins with a thorough psychiatric interview for diagnostic evaluation and treatment planning [35]. Stress and trauma are etiological factors in both SUD and ED, and diagnostic interviews must consider these aspects [12]. Treatments for ED and SUD are individualized, substance- or ED-specific, and mindful of an individual's intrinsic motivation and readiness for change [62]. For SUD, individuals identify abstinence or harm reduction goals; the latter refers to continued substance use instead of incrementally using less to mitigate risk 
Table 2 Prevalence of substance use disorders in AN, AN-R, and AN-BP

\begin{tabular}{|c|c|c|c|c|c|c|c|c|c|}
\hline $\begin{array}{l}\text { Substance use disorder (abuse/ } \\
\text { dependence) }\end{array}$ & $k$ & $n$ & $2^{2^{* *}}$ & $95 \% \mathrm{Cls}$ & $\begin{array}{l}\text { Prevalence } \\
\text { in AN }\end{array}$ & $\mathrm{n}$ for $\mathrm{AN}-\mathrm{R}$ & $\begin{array}{l}\text { Prevalence } \\
\text { in AN-R }\end{array}$ & $\mathrm{n}$ for AN-BP & $\begin{array}{l}\text { Prevalence } \\
\text { in AN-BP }\end{array}$ \\
\hline Alcohol & 25 & 12,655 & $84.08 \%$ & $0.08,0.13$ & $10 \%$ & 1752 & $3 \%$ & 1138 & $15 \%$ \\
\hline Cannabis & 6 & 1379 & $88.65 \%$ & $0.02,0.12$ & $6 \%$ & 551 & $0 \%$ & 461 & $4 \%$ \\
\hline Polysubstance & 3 & 1187 & & $0.01,0.04$ & $3 \%$ & 520 & $2 \%$ & 436 & $4 \%$ \\
\hline Amphetamine & 4 & 1203 & $92.79 \%$ & $0.00,0.15$ & $5 \%$ & 520 & $0 \%$ & 436 & $0 \%$ \\
\hline Sedative/hypnotic & 3 & 1190 & & $0.00,0.03$ & $1 \%$ & 582 & $0 \%$ & 472 & $1 \%$ \\
\hline Cocaine & 5 & 1280 & $83.02 \%$ & $0.00,0.08$ & $3 \%$ & 548 & $0 \%$ & 471 & $1 \%$ \\
\hline Hallucinogen & 2 & 1092 & & $0.00,0.01$ & $0 \%$ & 520 & $0 \%$ & 436 & $0 \%$ \\
\hline Opioid & $1^{*}$ & 956 & & $0.00,0.01$ & $0 \%$ & 520 & $0 \%$ & 436 & $0 \%$ \\
\hline Inhalant & 2 & 1054 & & $0.00,0.00$ & $0 \%$ & 582 & $0 \%$ & 472 & $0 \%$ \\
\hline Narcotic & $1^{*}$ & 136 & & $0.00,0.05$ & $1 \%$ & N/A & N/A & N/A & N/A \\
\hline Other substances & 4 & 1098 & $88.92 \%$ & $0.00,0.14$ & $4 \%$ & 551 & $0 \%$ & 461 & $0 \%$ \\
\hline Substance Use Disorder Total & 15 & 3118 & $86.3 \%$ & $0.11,0.20$ & $16 \%$ & 1635 & $7 \%$ & 1058 & $18 \%$ \\
\hline Drug Abuse/ Dependence Total & 13 & 10,443 & $81.1 \%$ & $0.05,0.09$ & $7 \%$ & 278 & $5 \%$ & 235 & $9 \%$ \\
\hline
\end{tabular}

${ }^{*}$ not derived from mete-analysis data, ${ }^{* *} \boldsymbol{I}^{2}$ only derived when pooled meta-analysis included 4 or more studies, $I^{2}$ for AN prevalence

$A N$ anorexia nervosa; $A N-B P$ anorexia nervosa binge-eating/purge type; $A N-R$ anorexia nervosa restrictive type; $C l s$ confidence intervals; $k$ amount of studies

Table 3 Prevalence of Substance Use in AN, AN-R, and AN-BP

\begin{tabular}{|c|c|c|c|c|c|c|c|c|c|}
\hline \multicolumn{10}{|l|}{ Prevalence } \\
\hline Substance Use & $k$ & $n$ & $1^{* *}$ & $95 \% \mathrm{Cls}$ & $\begin{array}{l}\text { Prevalence } \\
\text { in AN }\end{array}$ & $\mathrm{n}$ for $\mathrm{AN}-\mathrm{R}$ & $\begin{array}{l}\text { Prevalence in } \\
\text { AN-R }\end{array}$ & $n$ for AN-BP & $\begin{array}{l}\text { Prevalence } \\
\text { in AN-BP }\end{array}$ \\
\hline Alcohol & 5 & 677 & $70.56 \%$ & $0.22,0.36$ & $29 \%$ & 172 & $31 \%$ & 156 & $43 \%$ \\
\hline Amphetamines & 3 & 326 & & $0.01,0.06$ & $3 \%$ & N/A & N/A & N/A & N/A \\
\hline Barbiturates & 2 & 160 & & $0.00,0.05$ & $2 \%$ & N/A & N/A & N/A & N/A \\
\hline Caffeine & 3 & 107 & & $0.08,0.73$ & $37 \%$ & N/A & N/A & N/A & N/A \\
\hline Cannabis & 5 & 720 & $95.18 \%$ & $0.03,0.28$ & $14 \%$ & 283 & $16 \%$ & 211 & $33 \%$ \\
\hline Cocaine & 3 & 326 & & $0.00,0.04$ & $1 \%$ & 111 & $0 \%$ & 55 & $0 \%$ \\
\hline Diazepam & $1^{*}$ & 26 & & $0.00,0.20$ & $4 \%$ & N/A & N/A & N/A & N/A \\
\hline Hallucinogens & 2 & 161 & & $0.00,0.02$ & $0 \%$ & N/A & N/A & N/A & N/A \\
\hline Heroin & $1^{*}$ & 166 & & $0.00,0.02$ & $0 \%$ & N/A & N/A & N/A & N/A \\
\hline Inhalants & 2 & 148 & & $0.00,0.04$ & $1 \%$ & N/A & N/A & N/A & N/A \\
\hline Opiates & 3 & 521 & & $0.00,0.24$ & $5 \%$ & 111 & $1 \%$ & 55 & $2 \%$ \\
\hline Phencyclidine & $1^{*}$ & 26 & & $0.00,0.13$ & $0 \%$ & N/A & N/A & N/A & N/A \\
\hline Quaaludes & $1^{*}$ & 26 & & $0.00,0.20$ & $4 \%$ & N/A & N/A & N/A & N/A \\
\hline Stimulants & 2 & 383 & & $0.11,0.18$ & $14 \%$ & N/A & N/A & N/A & N/A \\
\hline Tobacco & 9 & 1352 & $90.64 \%$ & $0.16,0.34$ & $25 \%$ & 255 & $26 \%$ & 788 & $32 \%$ \\
\hline Tranquilizers & $1^{*}$ & 134 & & $0.00,0.06$ & $2 \%$ & N/A & N/A & N/A & N/A \\
\hline Other Substance Use & $1^{*}$ & 328 & & $0.10,0.18$ & $14 \%$ & N/A & N/A & N/A & N/A \\
\hline Substance Use Total & 4 & 769 & $94.27 \%$ & $0.08,0.34$ & $20 \%$ & 111 & $5 \%$ & 55 & $18 \%$ \\
\hline
\end{tabular}

* not derived from meta-analysis data, ${ }^{* *} \boldsymbol{I}^{2}$ only derived when pooled meta-analysis included 4 or more studies

$A N$ anorexia nervosa; $A N-B P$ anorexia nervosa binge-eating/purge type; $A N-R$ anorexia nervosa restrictive type; $C l s=$ confidence intervals; $k$ amount of studies

[31]. With these general frameworks in mind, the next component of treatment is usually tailored towards the specific ED-SUD combination. A variety of psychotherapeutic, psychosocial, and even pharmacological therapies are available for ED and SUD, but few studies have explored treatments for co-occurring disorders [6]. At present, clinicians generally pick treatments that could work synergistically, as some therapies have indications for both diseases. For alcohol use disorder (AUD), the three first-line medications are naltrexone (an opioid 
receptor antagonist blocking the endogenous reward associated with alcohol consumption), disulfiram (an inhibitor of acetaldehyde dehydrogenase, causing a toxic reaction if alcohol is consumed due to accumulation of alcohol metabolites), and acamprosate (an NMDA receptor antagonist that reduces cravings for alcohol). For opioid use disorder (OUD), first-line pharmacotherapies include methadone and buprenorphine, synthetic opioids that suppress opioid withdrawal and cravings [4, 32]. For tobacco use disorder (TUD), nicotine replacement therapy, varenicline (a partial agonist of the acetylcholinergic receptor), and bupropion (a noradrenergic-dopaminergic antidepressant) are evidence-based treatments that can improve quit rates and sustained abstinence from tobacco [37]. While the DSM-5 does not currently recognize caffeine use disorder, caffeine withdrawal and intoxication are formal diagnoses [1]. Generally, it is possible that treatments for co-occurring SUD in people with AN may not interfere with AN treatment.

Given the high degree of comorbidity between SUDs and AN, it is essential to develop treatment strategies that are effective for both conditions. Pharmacologically, most medications used to treat AN or to treat SUD are compatible with one another. For example, the use of opioid agonist therapies for opioid use disorder and naltrexone or acamprosate for alcohol use disorder could complement the pharmacological treatment. However, as some of these medications for SUDs can prolong the QTc interval, they raise the risk of new-onset cardiac arrhythmias, which may be more likely in persons with $\mathrm{AN}$ who are very underweight and if they have electrolyte abnormalities. There are currently no approved pharmacological interventions for stimulant, cannabis [5, 7], or hallucinogen use disorders. For cannabis use disorder (CUD), the occurrence of cannabis withdrawal and intoxication can also induce anorexia symptoms, nausea, vomiting, and weight loss and can occur in nearly half of persons with CUD [8]. For CUD, there are no approved pharmacotherapies, and the only treatment with current efficacy for withdrawal symptoms is sustained abstinence.

Nonetheless, several psychosocial interventions have evidence for both ED and co-occurring SUD, such as self-help approaches [59], mindfulness-based cognitive behavioral therapy [15], dialectical behavioral therapy [14], family and couples therapy [42], and contingency management [20]. While there are several reasons why some psychosocial interventions may demonstrate efficacy for co-occurring AN and SUD, one reason might be transdiagnostic psychopathology that responds to the same types of treatments [36]. For example, Claudat et al. made a case for the effectiveness of DBT for persons with co-occurring EDs and SUD as such persons share difficulties with emotion regulation, goal-directed activity, and impulsivity [14].

New and innovative interventions such as real-time fMRI-based neurofeedback, transcranial magnetic stimulation, transcranial direct current stimulation, and deep brain stimulation aim to influence brain regions' activity to regulate food and addictive substance intake [17]. These interventions follow the assumption that there are two circuits in the brain controlling both food intake in EDs and substance use in SUDs; the first circuit responds to salient/rewarding stimuli and consists of structures like ventral striatum, amygdala, anterior insula, ventromedial prefrontal cortex/orbitofrontal cortex. The second circuit regulates the degree of cognitive control over food or addictive substance intake and includes brain structures such as the anterior cingulum and dorsolateral prefrontal cortex [58]. Thus, future research in the form of randomized control trials are needed to test the variety of treatment strategies mentioned above in an effort to reduce symptoms in individuals suffering from comorbid SUDs and AN.

\section{Overlapping features between SUDs and AN}

Although SUD and AN potentially appear to respond to some of the same treatments, these disorders are distinct. However, both conditions are behaviourally defined psychiatric disorders [46] and may also have some genetic overlap [41]. For SUD, aberrations in the endogenous reward system drive pathological craving and drug-seeking behaviors, leading to a continued cycle of intoxication and withdrawal [2]. For AN, a negative view of one's body image drives caloric restriction, low BMI, and for some, repeated compensatory behaviors to maintain low body weight, such as purging, extreme exercise, laxative use, and fasting, often associated with subjective and objective binge episodes [1]. Several working hypotheses have been put forward as potential explanations for their overlap as these two disorders commonly co-occur [25]. The first hypothesis involves self-regulation through self-medication, which means that substance use and AN behavior (e.g., restriction, binge behaviors, purging) are used to "treat" an underlying pathology [30, 52]. Frequently, persons with SUD and AN both describe a chaotic inner milieu that temporarily abates from the effects of substances or caloric restriction [9]. The second hypothesis assumes shared risk factors or underlying causes. For example, a drive towards perfectionism, impulsivity, novelty-seeking, and rigidity/obsessions appear to raise the risk for the development of both ED and SUD [38, 53]. One of our review's findings -that the prevalence of substance use and SUD was higher in AN-BP compared to AN-R- appears to support this second hypothesis, as AN-BP has more features consistent with the impulsive, 
novelty-seeking phenotype seen in persons with SUD $[11,43,50]$. Finally, specific substances may appear to serve a functional purpose for forms of ED. In the setting of ED, appetite-suppressing substances, such as tobacco, may help maintain a low appetite $[3,40]$. In addition, caffeine and stimulants may also suppress appetite, maintain caloric restriction, fuel intense exercise, and address fatigue stemming from diminished BMI [10, 55]. Alcohol may lessen the severity of AN-induced anxiety and affective symptoms [13] and may increase appetite [51].

Recent neurobiological findings support the notion that mechanisms of addiction may also be involved in the development and maintenance of AN [44, 61]. On the one hand, high-calorie food cues, which may be considered "incompatible" with AN, are processed with anxiety and associated with increased activation of brain regions responsible for inhibitory control [61], which is per avoidance bias regarding food found in individuals with AN [45]. On the other hand, disorder-compatible stimuli (e.g., images of underweight women's bodies, physical activity cues) are appetitively processed; such sensitization processes of the reward system may lead to maintaining the problematic behavior patterns seen in AN. For example, females with AN instructed to imagine that their own body would correspond to specific normalweight or underweight body cues showed more robust activation in structures of the reward system, particularly in the ventral striatum, during the self-referential processing of images of underweight bodies compared with normal-weight bodies; the opposite pattern was found for healthy female subjects [23]. Similar results were shown using other techniques as well: both EEG and eye-tracking studies, as well as studies in which the blink reflex was recorded as a measure of appetitive valence, have revealed an attentional bias/positive processing for images of underweight female bodies and images of physical activity [27], comparable to the processing of alcohol-associated stimuli in alcohol-dependent patients. Accordingly, O'Hara and colleagues [44] postulated a "reward-centered" model of AN, which assumes that food-associated stimuli are experienced as aversive.

In contrast, disorder-compatible stimuli (such as underweight body images and physical activity) are processed positively and activate the mesolimbic reward system. Also, individuals with AN exhibit greater activation in prefrontal brain areas, somatosensory cortex, and cerebellum when responses to physical activity stimuli are to be inhibited in a go-no-go task [33]. This also corresponds to findings regarding the neuronal activation patterns of alcohol-dependent patients during response inhibition towards alcohol-associated stimuli. Such findings suggest an inhibition deficit for disorder-compatible rewarding behaviors.

\section{Strengths and limitations}

The present meta-analysis has several strengths. The robust methods and adherence to PRESS, PRISMA, and MOOSE guidelines are one, while the large yield of studies $(\mathrm{n}=52)$ and participants $(\mathrm{n}=14,695$ individuals identified as having AN) were others. In addition, the studies included in this meta-analysis were of fair to moderate quality. The review also advances the field by focusing on the prevalence of both substance use and SUD in persons with AN. While a previous review by Bahji et al. found similar SUD prevalence estimates in EDs [6], the present study identified more studies. While having SUD estimates provides a meaningful assessment of clinically significant impairment, the additional information provided by substance use helps contextualize the specific patterns of substances that are more likely to lead to functional consequences in persons with AN.

However, there are a few limitations. First, as a metaanalysis of prevalence, we encountered high heterogeneity when pooling estimates across studies. Some of this heterogeneity occurred from combining the different types of AN, and stratification into AN-BP and AN-Rspecific estimates helped reduce some heterogeneity. However, there are other potential sources of heterogeneity that we did not explore analytically due to the limited number of studies per subgroup analysis. For example, variations in substance use and SUD measurements across studies likely increased heterogeneity due to different ascertainment methods (e.g., self-report, informant-report, structured interviews, urine drug screens) and alternative diagnostic criteria (e.g., DSMIII, DSM-IV, and DSM-5). In addition, many specific substance use disorders and different types of substance use had a very low prevalence in those with $\mathrm{AN}$, however this may be due to the limited amount of studies available for these outcomes. Specifically, many of the substance use prevalence rates were much lower than the general populations, such as alcohol use and caffeine use. The generalizability of the results is another limitation, as most participants were young women; consequently, our review's findings are less applicable to males in general and older populations. Finally, we cannot determine causal relationships between substance use, SUD, and $\mathrm{AN}$ as an observational review.

\section{Conclusions}

This is the most comprehensive meta-analysis on the comorbid prevalence of SUDs and substance use in persons with AN, with an overall pooled prevalence of $16 \%$. Comorbid SUDs were much more common in AN-BP compared to AN-R. Clinicians should be aware of the high prevalence of specific SUD comorbidity and substance use in individuals with AN. Finally, clinicians 
should consider screening for SUDs and integrating treatments that target SUDs in individuals with AN.

\begin{abstract}
Abbreviations
AN-R: Anorexia nervosa-restricting type; AN-P: Anorexia nervosa-purging type; AN-B: Anorexia nervosa-binging type; ANBN: Anorexia nervosa and bulimia nervosa; ED: Eating disorder; AN-BP: Anorexia nervosa, binge-eating/purging type; BN: Bulimia nervosa; MDD: Major depressive disorder; AUD: Alcohol use disorder; DUD: Drug use disorder; SUG: Substance use group; NSUG: No substance use group.
\end{abstract}

\section{Supplementary Information}

The online version contains supplementary material available at https://doi. org/10.1186/s40337-021-00516-3.

Additional file 1. Supplementary Review Material.

\section{Acknowledgements}

This work was supported by the O'Brien Institute for Public Health \& Mathison Centre for Mental Health Postdoctoral Scholarship, the Cumming School of Medicine Post-Doctoral Scholarship, and the Harley Hotchkiss -Samuel Weiss Postdoctoral Fellowship awarded to Daniel Devoe. As well, the Cuthbertson \& Fischer Chair in Pediatric Mental Health was awarded to Scott Patten. This work is also supported by the Alberta Children's Hospital Foundation and the Alberta Children's Hospital Research Institute through Gina Dimitropoulos.

\section{Authors' contributions}

All authors edited and reviewed the manuscript. AA, JF, and AS helped screen titles and abstracts, and full text. In addition, they extracted the data for tables, figures, and the meta-analysis. TL conducted the risk of bias assessments. $A B$ wrote the discussion and GP wrote the introduction. GD and SP oversaw both the clinical and methodological aspects of the paper. DD conducted the meta-analysis and wrote the methods and results sections. All authors read and approved the final manuscript.

\section{Funding}

This work was supported by the O'Brien Institute for Public Health \& Mathison Centre for Mental Health Postdoctoral Scholarship, and the Cumming School of Medicine Post-Doctoral Scholarship awarded to Daniel Devoe.

\section{Availability of data and materials}

Not applicable.

\section{Declarations}

Ethics approval and consent to participate

Not applicable.

\section{Consent for publication}

All authors give their consent for publication.

\section{Competing interests}

The authors declare that they have no competing interests.

\author{
Author details \\ ${ }^{1}$ Department of Psychiatry, Cumming School of Medicine, Mathison Centre \\ for Mental Health Research and Education, University of Calgary, 3280 Hospital \\ Drive NW, Calgary, AB T2N 4Z6, Canada. ${ }^{2}$ Faculty of Social Work, University \\ of Calgary, Calgary, Canada. ${ }^{3}$ Ruhr-University Bochum, University Clinic for Psy- \\ chosomatic Medicine and Psychotherapy, Campus East-Westphalia, Lübbecke, \\ Germany.
}

Received: 22 July 2021 Accepted: 25 November 2021 Published online: 11 December 2021

\section{References}

1. American Psychiatric Association. DSM-V: Diagnostic and Statistical Manual of Mental Disorders, 5th Edition. (5th ed.). American Psychiatric Association (2013). https://doi.org/10.1176/appi.books.9780890425596

2. American Society of Addiction, M. Definition of Addiction (2021). Retrieved 2021/06/11/20:32:31 from https://www.asam.org/docs/default-source/ public-policy-statements/1definition_of_addiction_long_4-11.pdf? sfvrsn=a8f64512_4

3. Anzengruber D, Klump KL, Thornton L, Brandt H, Crawford S, Fichter MM, Halmi KA, Johnson C, Kaplan AS, LaVia M, Mitchell J, Strober M, Woodside DB, Rotondo A, Berrettini WH, Kaye WH, Bulik CM. Smoking in eating disorders. Eating Behav. 2006;7(4):291-9. https://doi.org/10.1016/j.eatbeh. 2006.06.005.

4. Bahji A, Bajaj N. Opioids on trial: a systematic review of interventions for the treatment and prevention of opioid overdose. Can J Addic. 2018; 9(1). https://journals.Iww.com/cja/Fulltext/2018/03000/Opioids_on_Trial__A_ Systematic_Review_of.4.aspx

5. Bahji A, Mazhar MN. Treatment of Cannabis dependence with synthetic cannabinoids: a systematic review. Can J Addic. 2016; 7(4). https://journ als.Iww.com/cja/Fulltext/2016/12000/Treatment_of_Cannabis_Depen dence_with_Synthetic.3.aspx

6. Bahji A, Mazhar MN, Hudson CC, Nadkarni P, MacNeil BA, Hawken E. Prevalence of substance use disorder comorbidity among individuals with eating disorders: a systematic review and meta-analysis. Psychiatry Res. 2019;273:58-66. https://doi.org/10.1016/j.psychres.2019.01.007.

7. Bahji A, Meyyappan AC, Hawken ER, Tibbo PG. Pharmacotherapies for cannabis use disorder: a systematic review and network meta-analysis. Int J Drug Policy. 2021;97, 103295. https://doi.org/10.1016/j.drugpo.2021. 103295

8. Bahji A, Stephenson C, Tyo R, Hawken ER, Seitz DP. Prevalence of cannabis withdrawal symptoms among people with regular or dependent use of cannabinoids. JAMA Netw Open. 2020;3(4), e202370. https://doi.org/10. 1001/jamanetworkopen.2020.2370

9. Barbarich-Marsteller NC, Foltin RW, Walsh BT. Does anorexia nervosa resemble an addiction? Curr Drug Abuse Rev. 2011;4(3):197-200.

10. Berardisa DD, Matarazzo I, Orsolini L, Aless VR, Tomasetti C, Montemitro C, Mazza M, Fornaro M, Carano R, Perna G, Vellante F, Sante DD, Rovere RL, Martinotti G, Trotta S, Giannantonio MD. The problem of eating disorders and comorbid psychostimulants abuse: a mini review. 2019;9(3):23592368. https://www.jneuropsychiatry.org/peer-review/the-problem-ofeating-disorders-and-comorbid-psychostimulants-abuse-a-mini-review. pdf

11. Bollen E, Wojciechowski FL. Anorexia nervosa subtypes and the big five personality factors. Eur Eating Disord Rev. 2004;12(2):117-21. https://doi. org/10.1002/erv.551.

12. Brewerton TD, Brady K. The role of stress, trauma, and PTSD in the etiology and treatment of eating disorders, addictions, and substance use disorders. In: Brewerton TD, Baker Dennis A, Brewerton TD, Baker Dennis A (eds), Eating disorders, addictions and substance use disorders: research, clinical and treatment perspectives, pp. 379-404 (2014). https://doi.org/ 10.1007/978-3-642-45378-6_17

13. Bulik CM, Klump KL, Thornton L, Kaplan AS, Devlin B, Fichter MM, Halmi KA, Strober M, Woodside DB, Crow S, Mitchell JE, Rotondo A, Mauri M, Cassano GB, Keel PK, Berrettini WH, Kaye WH. Alcohol use disorder comorbidity in eating disorders: a multicenter study. J Clin Psychiatry. 2004;65(7):1000-6.

14. Claudat K, Brown TA, Anderson L, Bongiorno G, Berner LA, Reilly E, Luo T, Orloff N, Kaye WH. Correlates of co-occurring eating disorders and substance use disorders: a case for dialectical behavior therapy. Eat Disord. 2020;28(2):142-56. https://doi.org/10.1080/10640266.2020.1740913.

15. Courbasson CM, Nishikawa Y, Shapira LB. Mindfulness-action based cognitive behavioral therapy for concurrent binge eating disorder and substance use disorders. Eat Disord. 2010;19(1):17-33. https://doi.org/10. 1080/10640266.2011.533603.

16. Dalton B, Bartholdy S, Campbell IC, Schmidt U. Neurostimulation in clinical and sub-clinical eating disorders: a systematic update of the literature. Curr Neuropharmacol. 2018;16(8):1174-92. https://doi.org/10.2174/15701 59X16666180108111532. 
17. Dalton B, Campbell IC, Schmidt U. Neuromodulation and neurofeedback treatments in eating disorders and obesity. Curr Opin Psychiatry. 2017;30(6):458-73.

18. DerSimonian R, Laird N. Meta-analysis in clinical trials. Controll Clin Trials. 1986;7(3):177-88. https://doi.org/10.1016/0197-2456(86)90046-2.

19. Downs $\mathrm{SH}$, Black N. The feasibility of creating a checklist for the assessment of the methodological quality both of randomised and nonrandomised studies of health care interventions. J Epidemiol Commun Health. 1998;52(6):377-84. https://doi.org/10.1136/jech.52.6.377.

20. Dutra L, Stathopoulou G, Basden SL, Leyro TM, Powers MB, Otto MW. A meta-analytic review of psychosocial interventions for substance use disorders. Am J Psychiatry. 2008;165(2):179-87. https://doi.org/10.1176/ appi.ajp.2007.06111851.

21. Elmquist JA, Shorey RC, Anderson SE, Temple JR, Stuart GL. The relationship between eating disorder symptoms and treatment rejection among young adult men in residential substance use treatment. Substance Abuse Res Treat. 2016;10:39-44.

22. Fassino S, Pierò A, Tomba E, Abbate-Daga G. Factors associated with dropout from treatment for eating disorders: a comprehensive literature review. BMC Psychiatry. 2009; 9.

23. Fladung A-K, Grön G, Grammer K, Herrnberger B, Schilly E, Grasteit S, Wolf $\mathrm{RC}$, Walter $\mathrm{H}$, Von Wietersheim J. A neural signature of anorexia nervosa in the ventral striatal reward system. Am J Psychiatry. 2010;167(2):206-12. https://doi.org/10.1176/appi.ajp.2009.09010071.

24. Fladung AK, Schulze UME, Schöll F, Bauer K, Grön G. Role of the ventral striatum in developing anorexia nervosa. Transl Psychiatry. 2013:3(10):e315.

25. Fouladi F, Mitchell JE, Crosby RD, Engel SG, Crow S, Hill L, Le Grange D, Powers P, Steffen KJ. Prevalence of alcohol and other substance use in patients with eating disorders. Eur Eat Disord Rev J Eat Disord Assoc. 2015:23(6):531-6. https://doi.org/10.1002/erv.2410.

26. Godier LR, Park RJ. Compulsivity in anorexia nervosa: a transdiagnostic concept. Front Psychol. 2014;5:778.

27. Horndasch S, Kratz O, Van Doren J, Graap H, Kramer R, Moll GH, Heinrich $\mathrm{H}$. Cue reactivity towards bodies in anorexia nervosa - Common and differential effects in adolescents and adults. Psychol Med 2018;48(3):508-18.

28. Jansen JM, Daams JG, Koeter MWJ, Veltman DJ, van den Brink W, Goudriaan AE. Effects of non-invasive neurostimulation on craving: a metaanalysis. Neurosci Biobehav Rev. 2013;37(10, Part 2):2472-80. https://doi. org/10.1016/j.neubiorev.2013.07.009.

29. Keski-Rahkonen A, Mustelin L. Epidemiology of eating disorders in Europe: prevalence, incidence, comorbidity, course, consequences, and risk factors. Curr Opin Psychiatry. 2016;29(6):340-5. https://doi.org/10. 1097/yco.0000000000000278.

30. Khantzian EJ. The self-medication hypothesis of substance use disorders: a reconsideration and recent applications. Harvard Rev Psychiatry. 1997;4(5):231-44. https://doi.org/10.3109/10673229709030550.

31. Kleber, H. D. et al. Treatment of patients with substance use disorders, second edition. American Psychiatric Association. Am J Psychiatry. 2007;164(4 Suppl): 5-123.

32. Korownyk C, Perry D, Ton J, Kolber MR, Garrison S, Thomas B, Allan GM, Dugré N, Finley CR, Ting R, Yang PR, Vandermeer B, Lindblad AJ. Opioid use disorder in primary care: PEER umbrella systematic review of systematic reviews. Can Fam Phys. 2019;65(5):e194-206.

33. Kullmann S, Giel KE, Hu X, Bischoff SC, Teufel M, Thiel A, Zipfel S, PreissI H. Impaired inhibitory control in anorexia nervosa elicited by physical activity stimuli. Soc Cognit Affect Neurosci. 2014;9(7):917-23. https://doi.org/ 10.1093/scan/nst070.

34. Liberati A, Altman DG, Tetzlaff J, Mulrow C, Gotzsche PC, loannidis JP, Clarke M, Devereaux PJ, Kleijnen J, Moher D. The PRISMA statement for reporting systematic reviews and meta-analyses of studies that evaluate healthcare interventions: explanation and elaboration. BMJ. 2009;339:b2700. https://doi.org/10.1136/bmj.b2700.

35. Lingford-Hughes A, Welch S, Peters L, Nutt D. BAP updated guidelines: evidence-based guidelines for the pharmacological management of substance abuse, harmful use, addiction and comorbidity: recommendations from BAP. J Psychopharmacol. 2012;26(7):899-952. https://doi.org/ 10.1177/0269881112444324.

36. Luminet O, Bagby RM, Taylor GJ. Alexithymia: advances in research, theory, and clinical practice (2018)
37. Mills EJ, Wu P, Lockhart I, Thorlund K, Puhan M, Ebbert JO. Comparisons of high-dose and combination nicotine replacement therapy, varenicline, and bupropion for smoking cessation: a systematic review and multiple treatment meta-analysis. Ann Med. 2012;44(6):588-97. https://doi.org/10. 3109/07853890.2012.705016.

38. Minhas M, Murphy CM, Balodis IM, Acuff SF, Buscemi J, Murphy JG, MacKillop J. Multidimensional elements of impulsivity as shared and unique risk factors for food addiction and alcohol misuse. Appetite. 2021;159:105052. https://doi.org/10.1016/j.appet.2020.105052

39. Moher D, Shamseer L, Clarke M, Ghersi D, Liberati A, Petticrew M, Shekelle $P$, Stewart LA. Preferred reporting items for systematic review and metaanalysis protocols (PRISMA-P) 2015 statement. Syst Rev. 2015;4(1):1. https://doi.org/10.1186/2046-4053-4-1.

40. Mph NAG, Kimberly Frost-Pineda P, Md MSG. Tobacco and psychiatric dual disorders. J Addict Dis. 2007; 26(sup1):5-12. https://doi.org/10.1300/ J069v26S01_02

41. Munn-Chernoff MA, et al. Shared genetic risk between eating disorderand substance-use-related phenotypes: evidence from genome-wide association studies. Addict Biol. 2021;26(1):e12880. https://doi.org/10. 1111/adb.12880.

42. Murray SB, Labuschagne Z, Le Grange D. Family and couples therapy for eating disorders, substance use disorders, and addictions. In: Brewerton TD, Baker Dennis A, Brewerton TD, Baker Dennis A (eds) Eating disorders, addictions and substance use disorders: research, clinical and treatment perspectives, pp. 563-586 (2014). https://doi.org/10.1007/978-3-64245378-6_26

43. Nøkleby H. Comorbid drug use disorders and eating disorders - a review of prevalence studies. Nordic Stud Alcohol Drugs. 2012;29(3):303-14. https://doi.org/10.2478/v10199-012-0024-9.

44. O'Hara CB, Campbell IC, Schmidt U. A reward-centred model of anorexia nervosa: a focussed narrative review of the neurological and psychophysiological literature. Neurosci Biobehav Rev. 2015;52:131-52.

45. Paslakis G, Kühn S, Schaubschläger A, Schieber K, Röder K, Rauh E, Erim Y. Explicit and implicit approach vs. avoidance tendencies towards high vs. low calorie food cues in patients with anorexia nervosa and healthy controls. Appetite. 2016;107:171-9.

46. Peters ME, Taylor J, Lyketsos CG, Chisolm MS. Beyond the DSM: the perspectives of psychiatry approach to patients. Primary Care Companion CNS Disord. 2012. https://doi.org/10.4088/PCC.11m01233.

47. PRESS Peer Review of Electronic Search Strategies. Canadian Agency for Drugs and Technologies in Health (2016).

48. Rachid F. Neurostimulation techniques in the treatment of nicotine dependence: a review. Am J Addic. 2016;25(6):436-51. https://doi.org/10. 1111/ajad.12405.

49. Rachid F. Neurostimulation techniques in the treatment of cocaine dependence: a review of the literature. Addict Behav. 2018;76:145-55. https://doi.org/10.1016/j.addbeh.2017.08.004.

50. Root T, Pinheiro AP, Thornton L, Strober M, Fernandez-Aranda F, Brandt H, Crawford S, Fichter MM, Halmi KA, Johnson C, Kaplan AS, Klump KL, La Via M, Mitchell J, Woodside DB, Rotondo A, Berrettini WH, Kaye WH, Bulik CM. Substance use disorders in women with anorexia nervosa. Int J Eat Disord. 2010;43(1):14-21. https://doi.org/10.1002/eat.20670.

51. Rosager EV, Møller C, Sjögren M. Treatment studies with cannabinoids in anorexia nervosa: a systematic review. Eat Weight Disord EWD. 2021;26(2):407-15. https://doi.org/10.1007/s40519-020-00891-x.

52. Schoemaker C, Smit F, Bijl RV, Vollebergh WAM. Bulimia nervosa following psychological and multiple child abuse: support for the self-medication hypothesis in a population-based cohort study. Int J Eat Disord. 2002;32(4):381-8. https://doi.org/10.1002/eat.10102.

53. Schulte EM, Grilo CM, Gearhardt AN. Shared and unique mechanisms underlying binge eating disorder and addictive disorders. Clin Psychol Rev. 2016:44:125-39. https://doi.org/10.1016/j.cpr.2016.02.001.

54. StataCorp. Stata Statistical Software: Release 17. In StataCorp LLC (2021).

55. Striegel-Moore RH, Franko DL, Thompson D, Barton B, Schreiber GB, Daniels SR. Caffeine intake in eating disorders. Int J Eat Disord. 2006;39(2):162-5. https://doi.org/10.1002/eat.20216.

56. Stroup DF, Berlin JA, Morton SC, Olkin I, Williamson GD, Rennie D, Moher D, Becker BJ, Sipe TA, Thacker SB. Meta-analysis of observational studies in epidemiology: a proposal for reporting. Meta-analysis Of Observational Studies in Epidemiology (MOOSE) group. JAMA. 2000;283(15):2008-12. https://doi.org/10.1001/jama.283.15.2008. 
57. Umberg EN, Shader RI, Hsu LKG, Greenblatt DJ. From disordered eating to addiction: The "food drug" in bulimia nervosa. J Clin Psychopharmacol. 2012;32(3):376-89.

58. Val-Laillet D, Aarts E, Weber B, Ferrari M, Quaresima V, Stoeckel LE, AlonsoAlonso M, Audette M, Malbert CH, Stice E. Neuroimaging and neuromodulation approaches to study eating behavior and prevent and treat eating disorders and obesity. Neuroimage Clin. 2015:8:1-31.

59. von Ranson KM, Farstad SM. Self-help approaches in the treatment of eating disorders, substance use disorders, and addictions. In: Brewerton TD,Baker Dennis A, Brewerton TD, Baker Dennis A (eds) Eating disorders, addictions and substance use disorders: research, clinical and treatment perspectives, pp. 587-608 (2014). https://doi.org/10.1007/978-3-64245378-6_27

60. Walsh BT. The enigmatic persistence of anorexia nervosa. Am J Psychiatry 2013;170(5):477-84.

61. Wierenga CE, Ely A, Bischoff-Grethe A, Bailer UF, Simmons AN, Kaye WH. Are extremes of consumption in eating disorders related to an altered balance between reward and inhibition? Front Behav Neurosci. 2014;9(8):410.

62. Wilson GT, Shafran R. Eating disorders guidelines from NICE. Lancet. 2005;365(9453):79-81. https://doi.org/10.1016/S0140-6736(04)17669-1.

\section{Publisher's Note}

Springer Nature remains neutral with regard to jurisdictional claims in published maps and institutional affiliations.

- fast, convenient online submission

- thorough peer review by experienced researchers in your field

- rapid publication on acceptance

- support for research data, including large and complex data types

- gold Open Access which fosters wider collaboration and increased citations

- maximum visibility for your research: over $100 \mathrm{M}$ website views per year

At BMC, research is always in progress.

Learn more biomedcentral.com/submissions 